Pecvnia, Monográfico (2009), pp. 279-310

\title{
El impuesto sobre beneficios
}

\author{
Belén Morala Gómez \\ belen.morala@unileon.es \\ Universidad de León \\ Fac. de Ciencias Económicas y Empresariales \\ Campus de Vegazana, $\mathrm{s} / \mathrm{n}$ \\ 24071 León (España)
}

\section{INTRODUCCIÓN}

La concepción del impuesto sobre beneficios ha ido variando a lo largo del tiempo, desde la versión tradicional, según la cual el impuesto era uno más de los conceptos de reparto del resultado, hasta la versión actual que acepta sin discusión que el impuesto representa un gasto más del ejercicio.

Sobre la cuantificación de dicho gasto, hemos de tener en cuenta que el impuesto sobre beneficios grava la creación de riqueza por parte de la empresa. En el ámbito contable el cálculo de la riqueza se basa en criterios económicos. La Hacienda Pública, por su parte, calcula la riqueza generada en función de unos criterios, condicionados por la política fiscal y macroeconómica, que permitirán obtener la cuota a pagar. 
La contabilización del gasto por el impuesto de acuerdo con la cuota a pagar no está actualmente permitida en la mayoría de los sistemas contables, pues rompe con los principios de devengo y correlación de ingresos y gastos.

Por otra parte, la contabilización del gasto por impuesto de acuerdo con los principios de devengo y correlación de ingresos y gastos, el denominado método del efecto impositivo, exige presentar por separado lo que el sistema contable considera que debe ser el gasto por impuesto y el pago a la Haciendo Pública o cuota a pagar. La no coincidencia entre esas dos magnitudes plantea un nuevo problema, cómo es el relativo al tratamiento contable que debe otorgarse a las diferencias entre el gasto por impuesto y la cuota a pagar.

A lo largo del tiempo las distintas variantes del método del efecto impositivo han ido dando respuesta a este problema.

En primer lugar, dado que las diferencias a las que hacemos referencia, son consecuencia de la diferente consideración contable y fiscal de los sucesos económicos, existirían dos enfoques para la contabilización del impuesto:

a) Enfoque basado en la cuenta de resultados: considera las diferencias que se producen entre las valoraciones de los elementos de la cuenta de resultados, ingresos y gastos, a efectos contables y fiscales. Esas diferencias pueden ser temporales o permanentes, según reviertan o no en ejercicios futuros.

b) Enfoque basado en el balance: considera las diferencias entre las valoraciones contables y fiscales de los elementos del balance de situación. Se trata ahora de las llamadas diferencias temporarias.

En segundo lugar, podrían reconocerse o no de forma explícita los activos y pasivos fiscales motivados por las anteriores diferencias. Actualmente, resulta generalmente aceptado su reconocimiento explícito.

En tercer lugar, el reconocimiento explícito podría afectar a todas las diferencias o sólo a algunas de ellas. En el caso de las diferencias que dan lugar a activos, es evidente que algunas no podrán reconocerse contablemente, al no cumplir las condiciones de prudencia que exige su inclusión en el activo. La opción surge, por lo tanto, únicamente en relación con las diferencias que originan pasivos. La justificación para su reconocimiento parcial estriba en que algunas de las diferencias que 
originan dichos pasivos pueden ser recurrentes, por lo que el mayor pago futuro de impuestos puede no producirse en un futuro previsible. No obstante, también esta posibilidad se encuentra superada en la actualidad; la mayoría de las normativas contables exigen el reconocimiento total de las diferencias.

Por último, podría emplearse el método de capitalización o el método de la deuda. La diferencia entre ambos radica en la posibilidad o no, de ajustar los activos y pasivos impositivos que figuran en el balance en función de los cambios en los tipos de gravamen. Esta posibilidad no existe en el primer caso (se trata de no contaminar los resultados de periodos siguientes), mientras que en el segundo, se considera necesario el ajuste continuo de dichos saldos en función del tipo de gravamen esperado en el momento de su realización. El objetivo es ahora la presentación correcta de los activos y pasivos que aparecen en el balance de situación, aunque tales ajustes afecten al resultado de un periodo que no se corresponde ni con la aparición ni con la reversión de las diferencias.

La contabilización del impuesto de beneficios en España sigue actualmente el enfoque de la deuda basado en el balance.

\section{MÉTODO DE LA DEUDA BASADO EN EL BALANCE: LAS} DIFERENCIAS TEMPORARIAS

Las diferencias temporarias son las derivadas de la diferente valoración, contable y fiscal, atribuida a los activos, pasivos y determinados instrumentos de patrimonio de la empresa en la medida en que tengan incidencia en la carga fiscal futura.

De acuerdo con el PGC, la base fiscal de activos y pasivos coincide con la valoración fiscal de dichos elementos patrimoniales, es decir, con el importe atribuido a los mismos de acuerdo con la legislación fiscal aplicable.

Dado que la anterior definición resulta poco operativa desde un punto de vista contable, la NIC 12, profundizando más en esta cuestión, define la base fiscal de un activo como el importe deducible fiscalmente de los beneficios futuros obtenidos de dicho activo cuando se recupere su valor en libros. Si tales beneficios económicos no tributan, la base fiscal de los activos será igual a su valor en libros. 
Se corresponde, por lo tanto, con "lo deducible" fiscalmente en ejercicios futuros.

La base fiscal de un pasivo coincide con el valor contable del pasivo menos cualquier importe que resulte deducible fiscalmente respecto de tal partida en ejercicios futuros. En el caso de ingresos anticipados, la base fiscal es su importe en libros menos cualquier importe que no resulte imponible en periodos futuros. En este caso, la base fiscal se asocia a "lo no deducible" fiscalmente en un futuro.

Con respecto al tema de la base fiscal, es preciso tener en cuenta que se habla de base fiscal de activos y pasivos, pero no de la base fiscal de los elementos del patrimonio neto. Esto es así, porque estas últimas partidas se definen de forma residual, como diferencia entre activos y pasivos, por lo que su base fiscal no resulta relevante.

Por otra parte, también es necesario considerar el hecho de que, en ocasiones, existen partidas que no aparecen contabilizadas como activos o pasivos en el balance de situación y, sin embargo, poseen base fiscal. Ejemplos de este tipo de situaciones serían los activos totalmente amortizados a efectos contables, que siguen teniendo base fiscal o partidas contablemente reconocidas como gastos pero que, a efectos fiscales, son activos.

En otras ocasiones la dificultad para determinar la base fiscal es la condición establecida para reconocer una diferencia temporaria: que la misma tenga incidencia en la carga fiscal futura. Desde este punto de vista, las diferencias de carácter permanente no pueden considerarse diferencias temporarias.

\section{Ejemplo $\mathbf{n}^{0}$ 1.- Diferencias permanentes}

Una sociedad refleja en su balance al final de 2008 un pasivo de 1.000 u.m. como consecuencia de unas donaciones que realiza a entidades privadas y que fiscalmente están consideradas como gastos no deducibles.

Solución.-

Valor contable del pasivo: 1.000 u.m.

Base fiscal del pasivo $=$ valor contable - importe fiscalmente deducible en el futuro $=$ $1.000-0$ (no existen repercusiones fiscales futuras) $=1.000$ u.m.

Diferencia temporaria: valor contable del pasivo - base fiscal del pasivo $=0$ u.m., la diferencia permanente no tiene, como podemos comprobar, carácter de diferencia temporaria. 
Finalmente, las anteriores definiciones relativas a la base fiscal de activos y pasivos ponen de manifiesto que su cuantificación va depender de la forma en que se espera recuperar el activo o cancelar el pasivo.

Existen dos tipos de diferencias temporarias:

- Diferencias temporarias imponibles: son aquellas que dan lugar a mayores cantidades a pagar o menores cantidades a devolver en ejercicios futuros, a medida que se recuperen los activos o se liquiden los pasivos de los que se derivan.

- Diferencias temporarias deducibles: son aquellas que dan lugar a menores cantidades a pagar o mayores cantidades a devolver por impuestos en ejercicios futuros, a medida que se recuperen los activos o se liquiden los pasivos de los que se derivan.

El mayor o menor pago de impuestos en el futuro consecuencia de las diferencias temporarias se reconocerá, respectivamente, como:

- Un pasivo por impuesto diferido,

- Un activo por impuesto diferido.

Los importes de dichos activos y pasivos se calcularán aplicando el tipo de gravamen a la correspondiente diferencia temporaria.

Presentamos, a continuación, una serie de ejemplos relativos al cálculo de las bases contables y fiscales de activos y pasivos, diferencias temporarias y activos y pasivos por impuestos diferidos.

\section{Ejemplo n ${ }^{0}$ 2.-}

La sociedad X adquirió durante el ejercicio una máquina por 10.000 u.m. A la fecha del balance su amortización acumulada es de 1.000 u.m.

A esa misma fecha, se ha deducido fiscalmente una amortización de 1.500. El resto será deducible en ejercicios futuros.

El tipo de gravamen aplicable es del $30 \%$.

\section{Solución.-}

Valor en libros de la máquina al cierre del ejercicio: $10.000-1.000=9.000$ u.m. 
Base fiscal de la máquina: 8.500 u.m., ya que esa es la cantidad que será deducible en ejercicios futuros vía amortización (si la máquina se sigue utilizando) o como coste de la misma (si la máquina se vende).

Diferencia temporaria: $9.000-8.500=500$, será imponible, porque dará lugar a un mayor pago por impuestos en el futuro. En efecto, se prevé recuperar 9.000 u.m. de la máquina, de las que solo resultarán fiscalmente deducibles 8.500 u.m.

Pasivos por diferencias temporarias imponibles: $30 \% 500=150$ u.m., ésta será la cuantía del mayor pago de impuestos en el futuro derivado de la diferencia temporaria.

\section{Ejemplo n ${ }^{0}$ 3.-}

En el balance figuran "Intereses a cobrar" por un importe de 500 u.m., intereses que al cierre están devengados pero pendientes de cobro.

En la jurisdicción fiscal correspondiente, los intereses tributan cuando se cobren.

El tipo de gravamen aplicable es del $30 \%$.

\section{Solución.-}

Valor contable de la partida intereses a cobrar: 500 u.m.

Base fiscal: 0, desde el punto de vista fiscal, el asiento relativo a los intereses aún no se ha producido. Por otra parte, cuando se cobren los 500, y se tribute por ellos, no se podrá deducir nada.

Diferencia temporaria: $500-0=500$ u.m., será imponible porque dará lugar a un mayor pago por impuestos en el futuro, cuando se recupere el valor en libros del activo.

Pasivos por diferencias temporarias imponibles: $30 \% 500=150$ u.m.

\section{Ejemplo n ${ }^{0}$ 4.-}

La partida de clientes tiene un saldo contable de 600 u.m., de las cuales 200 u.m. se consideran de difícil cobro, por lo que se efectúa la correspondiente corrección valorativa, con el consiguiente gasto contable.

Desde el punto de vista fiscal, el gasto originado por la corrección valorativa no será deducible hasta un ejercicio posterior.

El tipo de gravamen aplicable es del 30\%.

\section{Solución.-}

Valor contable del activo: $600-200=400$ u.m.

Base fiscal del activo: 200 u.m., que es la cantidad deducible en el próximo ejercicio.

Diferencia temporaria: $400-200=200$ u.m., que será deducible dado que dará lugar a un menor pago de impuestos en el futuro.

Activo por diferencias temporarias deducibles: $30 \% 200=60$ u.m. 


\section{Ejemplo n ${ }^{0}$ 5.-}

Una entidad tiene un activo por importe de 9.000 u.m., que se corresponde con el principal de un préstamo concedido a un cliente. La devolución del principal no tiene consecuencias fiscales.

El tipo de gravamen aplicable es del $30 \%$.

\section{Solución.-}

Valor contable del activo: 9.000 u.m.

Base fiscal: puesto que la recuperación del valor en libros no tiene consecuencias fiscales, la base fiscal del activo coincide con su valor en libros, es decir, 9.000 u.m..

Diferencia temporaria: 0

\section{Ejemplo n ${ }^{0}$ 6.-}

Con fecha 31-12-08, una sociedad tiene registrado un cliente por importe de 20.000 u.m. como consecuencia de una venta que fiscalmente será computada como ingreso en el año del cobro, el año 2010

El tipo de gravamen aplicable es del $30 \%$.

\section{Solución.-}

Valor contable del activo: 20.000 u.m.

Base fiscal del activo: 0, cuando se recupere el activo, no se podrá deducir nada.

Diferencia temporaria: 20.000 u.m., que será imponible, porque dará lugar a un mayor pago de impuestos en el futuro, cuando se recupere vía cobro, el valor contable del activo.

Pasivo por diferencias temporarias imponibles: $30 \% 20.000=6.000$ u.m.

\section{Ejemplo n ${ }^{0}$ 7.-}

Dentro de los pasivos del balance figura una provisión de 4.000 u.m., para atender garantías de los artículos vendidos.

Fiscalmente, en este ejercicio solo se ha admitido un gasto por este concepto, de 3.000. El resto será deducible en el próximo ejercicio.

El tipo de gravamen aplicable es del $30 \%$.

\section{Solución.-}

Valor contable del pasivo: 4.000 u.m.

Base fiscal del pasivo: valor contable-importe fiscalmente deducible en el futuro, 4.000 $1.000=3.000$ u.m. 
Diferencia temporaria: 1.000 , que será deducible, porque dará lugar a un menor pago de impuestos en el futuro, cuando dicho importe resulte fiscalmente deducible.

Activo por diferencias temporarias deducibles: $30 \% 1.000=300$ u.m.

\section{Ejemplo $n^{0}$ 8.-}

El balance de una sociedad a 31-12-08 recoge la existencia de una cuenta de pasivo por un importe de 5.000 u.m. que representa el ingreso anticipado por el cobro de una prestación de servicios que se realizará el próximo año. Dichos ingresos se imputan tanto contable como fiscalmente siguiendo el principio del devengo.

El tipo de gravamen aplicable es del $30 \%$.

\section{Solución.-}

Valor contable del pasivo: 5.000 u.m.

Base fiscal del pasivo: 5.000 - 0 (importe no imponible en el futuro) $=5.000$ u.m.

Diferencia temporaria: 0 , no existen diferencias entre el tratamiento contable y fiscal de la operación.

De acuerdo con el PGC las circunstancias que originan diferencias temporarias son las siguientes:

1) Diferencias temporales entre la base imponible y el resultado contable antes de impuestos como consecuencia de los diferentes criterios de imputación temporal de ingresos y gastos en el ámbito contable y fiscal.

\section{Ejemplo nº 9.-}

Una empresa adquiere a comienzos de 2008 unos equipos informáticos por valor de $500.000 €$, cuya vida útil se fija en 4 años, sin valor residual. Fiscalmente, dichos equipos informáticos tienen un coeficiente máximo de amortización del 20\%.

\section{Solución.-}

Estamos ante una diferencia de carácter temporal entre el resultado contable y la base imponible del impuesto ocasionada por los distintos criterios de amortización empleados contable y fiscalmente.

La evolución de dicha diferencia temporal será la siguiente: 


\begin{tabular}{|c|c|c|c|}
\hline Ejercicio & $\begin{array}{c}\text { Amortización } \\
\text { contable }\end{array}$ & $\begin{array}{c}\text { Amortización } \\
\text { fiscal }\end{array}$ & $\begin{array}{c}\text { Diferencia } \\
\text { temporal }\end{array}$ \\
\hline 2008 & 125.000 & 100.000 & +25.000 \\
\hline 2009 & 125.000 & 100.000 & +25.000 \\
\hline 2010 & 125.000 & 100.000 & +25.000 \\
\hline 2011 & 125.000 & 100.000 & +25.000 \\
\hline 2012 & & 100.000 & -100.000 \\
\hline
\end{tabular}

Podemos observar cómo las diferencias que aparecen en los cuatro primeros años se compensan totalmente en el año 5, algo razonable si pensamos que el gasto total por amortización es el mismo a efectos contables y fiscales, 500.000 u.m., la diferencia estriba en la distribución temporal de dicho gasto.

En cuanto a la diferencia temporaria, que calculamos por diferencia entre el valor contable de los equipos informáticos y su base fiscal, su evolución es la que se recoge en el cuadro que se presenta a continuación:

\begin{tabular}{|c|c|c|c|c|c|}
\hline Ejercicio & $\begin{array}{c}\text { Valor } \\
\text { contable }\end{array}$ & $\begin{array}{c}\text { Base } \\
\text { fiscal }\end{array}$ & $\begin{array}{c}\text { Diferencia } \\
\text { temporaria } \\
\text { deducible total }\end{array}$ & $\begin{array}{c}\text { Diferencia } \\
\text { temporaria } \\
\text { del ejercicio }\end{array}$ & $\begin{array}{c}\text { Activo por } \\
\text { impuesto } \\
\text { diferido }\end{array}$ \\
\hline 2008 & 375.000 & 400.000 & 25.000 & 25.000 & 7.500 \\
\hline 2009 & 250.000 & 300.000 & 50.000 & 25.000 & 15.000 \\
\hline 2010 & 125.000 & 200.000 & 75.000 & 25.000 & 22.500 \\
\hline 2011 & 0 & 100.000 & 100.000 & 25.000 & 30.000 \\
\hline 2012 & & 0 & 0 & $(100.000)$ & 0 \\
\hline
\end{tabular}

La diferencia temporaria es de carácter deducible. Así por ejemplo, a finales de 2008, la base fiscal de los activos es de 400.000 (coste de 500.000 menos la amortización fiscal acumulada de 100.000). Para recuperar el valor contable de 375.000, la empresa debe obtener ganancias fiscales por 375.000, pero podrá deducir una amortización fiscal de 400.000. Como consecuencia, la empresa pagará menos impuestos en el futuro por valor de 7.500 (30\% de 25.000), a medida que vaya recuperando el importe en libros del activo. Ese menor impuesto se reconoce, a finales de 2008, como un activo por impuestos diferidos.

2) Ingresos y gastos imputados directamente al patrimonio neto que no se computan en la B.I., incluidas las variaciones de valor de los activos y pasivos, siempre que dichas variaciones difieran de las atribuidas a efectos fiscales.

\section{Ejemplo n ${ }^{0}$ 10.-}

Una sociedad posee ciertas participaciones en el capital social de otra sociedad, que se han clasificado como activos financieros disponibles para la venta. Fiscalmente, las posibles plusvalias tributan en el ejercicio en el que se enajenan las participaciones. (LIS: la revalorización no integrada en la BI no determina mayor valor, a efectos fiscales, de los elementos revalorizados).

Supongamos que el coste inicial de las acciones ha sido 20.000 y la evolución del valor razonable la siguiente: 
- 31-12-08: 22.000

- 31-12-09: 21.000

\section{Solución.-}

De acuerdo con la norma de valoración $\mathrm{n}^{\circ} 9$ del PGC, los activos financieros disponibles para la venta se valoran inicialmente por su valor razonable. La valoración posterior será también por su valor razonable y los cambios en dicho valor se registrarán directamente en el patrimonio neto hasta que el activo financiero cause baja en el balance o se deteriore. Así pues, se van a producir diferencias entre el valor contable de este tipo de activos (el valor razonable al cierre del ejercicio) y su base fiscal (el precio de la transacción o valor razonable inicial). En este caso, la diferencia temporaria que surge a finales de 2008 es imponible, cuando se recupere el valor contable del activo (22.000) habrá que pagar mayores impuestos por importe de 600 u.m. (30\% de 2.000), pues fiscalmente sólo podrán deducirse 20.000 en concepto de coste del activo.

\begin{tabular}{|c|c|c|c|c|c|}
\hline Ejercicio & $\begin{array}{c}\text { Valor } \\
\text { contable }\end{array}$ & $\begin{array}{c}\text { Base } \\
\text { fiscal }\end{array}$ & $\begin{array}{c}\text { Diferencia } \\
\text { temporaria } \\
\text { imponible total }\end{array}$ & $\begin{array}{c}\text { Diferencia } \\
\text { temporaria } \\
\text { del ejercicio }\end{array}$ & $\begin{array}{c}\text { Pasivo por } \\
\text { impuesto } \\
\text { diferido }\end{array}$ \\
\hline 2008 & 22.000 & 20.000 & 2.000 & 2.000 & 600 \\
\hline 2009 & 21.000 & 20.000 & 1.000 & $(1.000)$ & 300 \\
\hline
\end{tabular}

\section{3) En una combinación de negocios cuando los elementos patrimoniales se registran por un valor contable que difiere del atribuido a efectos fiscales.}

\section{Ejemplo $n^{0}$ 11.-}

La sociedad F adquiere a 31-12-08, la totalidad del patrimonio de otra sociedad, M cuyo balance de situación en ese momento es el siguiente:

\begin{tabular}{|c|c|c|c|}
\hline ACTIVO & & P. NETO Y PASIVO & \\
\hline 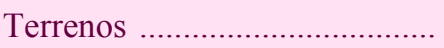 & 185.440 & 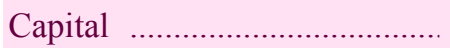 & 124.960 \\
\hline 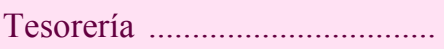 & 249.920 & Reservas .................. & 187.440 \\
\hline \multirow[t]{2}{*}{ Derechos de cobro ................... } & 2.000 & Deudas a $1 / p$ & 120.000 \\
\hline & & Deudas a $\mathrm{c} / \mathrm{p} \ldots$ & 4.960 \\
\hline Total & 437.360 & Total & 437.360 \\
\hline
\end{tabular}

El importe pagado por la sociedad F a los socios de M asciende a 413.930 u.m.

En el balance de $M$, el único elemento que difiere de su valor contable son los terrenos, que tienen un valor de 247.000 u.m.

Fiscalmente se tiene la siguiente información:

- El tipo impositivo es el 30\%. 
- La revalorización de elementos patrimoniales no tiene efectos fiscales.

- El fondo de comercio es fiscalmente deducible en un plazo máximo de 20 años.

\section{Solución.-}

Analizamos, en primer lugar, la diferencia temporaria que surge con respecto al terreno, cuyo valor contable pasa a ser 247.000 u.m., sin que tal revalorización tenga efectos fiscales:

Valor contable del activo: 247.000 u.m.

Base fiscal del activo: 185.440 u.m.

Diferencia temporaria imponible: $247.000-185.440=61.560$ u.m.

Pasivo por diferencias temporarias imponibles: 30\% 61.560 = 18.468 u.m.

Calculamos, a continuación, el valor del fondo de comercio. A tal efecto, hemos de tener en cuenta, además del nuevo valor contable de los terrenos, la aparición del pasivo por diferencias temporarias imponibles asociado a dichos terrenos:

Importe pagado por la adquisición del patrimonio de M: 413.930 u.m.

Valor razonable del patrimonio de $M=$ Valor razonable de los activos adquiridos - Valor razonable de los pasivos asumidos $=247.000+249.920+2.000-(120.000+4.960$ $+18.468)=355.492$ u.m.

Valor fondo de comercio: $413.930-355.492=58.438$ u.m.

Base fiscal del activo: 58.438 u.m. (es íntegramente deducible a efectos fiscales).

No existe, por lo tanto, diferencia temporaria alguna asociada al fondo de comercio.

\section{4) Reconocimiento inicial de un elemento, que no procede de una combinación de negocios y cuyo valor contable difiere del atribuido a efectos fiscales.}

\section{Ejemplo n ${ }^{0}$ 12.-}

El 1-1-08 una empresa adquiere un vehículo por 500.000 u.m. Su vida útil se estima en 5 años. Su amortización no es fiscalmente deducible.

\section{Solución.-}

Calculamos la diferencia temporaria que surge en el momento de reconocimiento inicial del vehículo:

Valor contable: 500.000 u.m.

Base fiscal: 0 (fiscalmente no podrá deducirse importe alguno en el futuro)

Diferencia temporaria imponible: 500.000 u.m.

Pasivo por diferencias temporarias imponibles: $30 \% 500.000=150.000$ u.m. 


\section{PASIVOS POR IMPUESTOS DIFERIDOS}

La norma de valoración $n^{\circ} 13$ del PGC, en consonancia con lo dispuesto por la NIC 12, establece que se reconocerán pasivos por impuestos diferidos por todas las diferencias temporarias imponibles salvo que tales pasivos surjan de:

a) Reconocimiento inicial de un fondo de comercio.

En los casos en los que el importe del fondo de comercio no sea fiscalmente deducible, surge en el momento de su reconocimiento una diferencia temporaria imponible entre su importe en libros y su base fiscal (lo deducible fiscalmente en el futuro) nula.

No se permite el reconocimiento del pasivo por impuestos diferidos correspondiente, pues el fondo de comercio se valora de forma residual, por lo que el reconocimiento de un pasivo de esta naturaleza incrementaría su propio valor en libros.

Evidentemente, aunque el PGC no lo mencione, las reducciones posteriores en un pasivo por impuestos diferidos que no se ha reconocido contablemente por proceder del reconocimiento inicial del fondo de comercio, se considera que también son consecuencia de dicho reconocimiento inicial y, por lo tanto, no se contabilizan (apartado 21 de la NIC 12).

b) Reconocimiento inicial de un activo o un pasivo en una transacción que no es una combinación de negocios y además no afectó ni al resultado contable ni a la base imponible del impuesto.

Como explicamos anteriormente, las diferencias temporarias pueden aparecer al registrar por primera vez un activo o un pasivo, cuando una parte o la totalidad de su valor no son deducibles a efectos fiscales.

Así, en el caso propuesto en el ejemplo 12, aparecía una diferencia temporaria imponible a la que correspondía un pasivo por diferencias temporarias imponibles de 150.000 u.m. Si tal pasivo se reconociera, dado que la operación no ha afectado al resultado contable ni a la base imponible del impuesto, debería ajustarse el importe en libros del activo, lo que volvería menos transparentes los estados financieros. 
En la misma línea de lo apuntado en el epígrafe anterior, las empresas no reconocerán tampoco, a medida que el activo se amortice, los cambios en el activo o pasivo por impuestos diferidos que no hubieran reconocido con anterioridad.

c) Inversiones en empresas dependientes, asociadas y negocios conjuntos si la inversora puede controlar el momento de la reversión de la diferencia y, además, es probable que tal diferencia no revierta en un futuro previsible.

Las diferencias temporarias aparecen cuando el importe en libros de las inversiones financieras en dependientes, sucursales y asociadas, o de las participaciones en negocios conjuntos, difiere de su valor fiscal. La empresa debe reconocer un pasivo por impuestos diferidos en todos los casos de diferencias temporarias asociadas con inversiones en dependientes, sucursales y negocios conjuntos, excepto cuando se den conjuntamente las dos condiciones siguientes:

- La dominante o inversora es capaz de controlar el momento de reversión de la diferencia temporaria.

- Es probable que la diferencia temporaria no revierta en un futuro previsible.

Si la sociedad dominante tiene poder para establecer la política de dividendos de su dependiente, también podrá controlar el momento de reversión de las diferencias temporarias asociadas con la inversión (procedentes de beneficios no distribuidos o diferencias de conversión). Normalmente, será muy difícil estimar la cuantía de los impuestos a pagar cuando tales diferencias reviertan. Así pues, cuando la dominante estime que tales beneficios no van a ser objeto de distribución en un futuro próximo, no se reconocerá el pasivo por diferencias temporarias imponibles.

Por el contrario, una empresa que invierte en una asociada no controla a esa empresa, ni puede determinar su política de dividendos. Por lo tanto, sin un acuerdo que establezca que la asociada no va a distribuir dividendos en un futuro próximo, la inversora reconocerá un pasivo por diferencias temporarias imponibles. 


\section{ACTIVOS POR IMPUESTOS DIFERIDOS}

Los activos por impuestos diferidos pueden surgir como consecuencia de cualquiera de las tres situaciones siguientes:

- Por diferencias temporarias deducibles.

- Por el derecho a compensar en ejercicios posteriores pérdidas fiscales.

- Por deducciones y ventajas fiscales pendientes de aplicar fiscalmente.

Para que estos activos puedan ser reconocidos contablemente, de acuerdo con el principio de prudencia, debe resultar probable que la empresa disponga de ganancias fiscales futuras que permitan su aplicación. En efecto, hay que tener en cuenta que la reversión de los anteriores activos va a dar lugar a una reducción en el importe de los beneficios fiscales de ejercicios posteriores. Pues bien, la empresa solo obtendrá beneficios económicos, en forma de reducciones de impuestos, cuando sea capaz de obtener ganancias fiscales suficientes en esos ejercicios futuros como para cubrir las posibles deducciones.

El PGC no añade ninguna consideración sobre la aplicación del principio de prudencia, no obstante, la NIC12, analizando la cuestión más en profundidad, realiza las siguientes precisiones:

1) Será probable que existan beneficios fiscales futuros, siempre que la empresa disponga de diferencias temporarias imponibles en cuantía suficiente, relacionadas con la misma autoridad fiscal y cuya reversión se vaya a producir:

a) en el mismo ejercicio fiscal en el que prevea que reviertan las diferencias temporarias deducibles, o bien,

b) en los ejercicios en los que se pueda compensar una pérdida fiscal o, por extensión, aplicar las deducciones y ventajas fiscales pendientes.

2) Cuando la cuantía de las diferencias temporarias imponibles sea insuficiente, solo se reconocerán activos por impuestos diferidos si se produce alguno de las situaciones siguientes:

a) Es probable que la empresa vaya a tener suficientes ganancias fiscales en el ejercicio en que reviertan las diferencias temporarias deducibles, se puedan compensar las 
pérdidas fiscales o aplicar las deducciones y demás ventajas fiscales.

b) La empresa tiene la posibilidad de aprovechar oportunidades de planificación fiscal para crear ganancias fiscales en los ejercicios oportunos.

Con respecto al caso concreto del reconocimiento de activos por impuestos diferidos que nacen de la posibilidad de compensar pérdidas y ventajas fiscales no aplicadas, la NIC 12 añade que la existencia de pérdidas fiscales no compensadas puede ser una evidencia para suponer que, en el futuro, no se dispondrá de beneficios fiscales. Por lo tanto, una empresa con un historial de pérdidas recientes solo reconocerá activos por impuestos diferidos procedentes de pérdidas o ventajas fiscales no aplicadas, en la medida en la que disponga de un importe suficiente de diferencias temporarias imponibles, o bien, cuando exista alguna otra evidencia convincente de que dispondrá en el futuro de suficientes ganancias fiscales contra las que cargar dichas pérdidas o ventajas fiscales.

Las excepciones a este criterio de reconocimiento de los activos por impuestos diferidos, en línea con lo ya apuntado en el caso de los pasivos por impuestos diferidos, son las que se enuncian a continuación:

a) Cuando la diferencia temporaria haya surgido por el reconocimiento inicial de un activo o un pasivo en una transacción que no sea una combinación de negocios y además, no afectó ni al resultado contable ni a la base imponible del impuesto.

b) Cuando la diferencia temporaria deducible haya surgido por inversiones en empresas dependientes, asociadas o negocios conjuntos, sólo se reconocerá un activo por impuesto diferido si se espera que dicha diferencia revierta en un futuro previsible y sea probable que la empresa disponga de ganancias fiscales futuras en cuantía suficiente.

Por otra parte, a la fecha de cierre de cada ejercicio la empresa tendrá que reconsiderar los activos por impuestos diferidos reconocidos contablemente, así como aquellos otros que no hubiera reconocido con anterioridad. La finalidad será dar de baja aquellos activos 
reconocidos cuya recuperación no se considera ya probable o reconocer aquellos otros que inicialmente no cumplían las condiciones, pero para los que a la fecha de cierre, resulte probable que la empresa disponga de ganancias fiscales futuras que permitan su aplicación.

\section{VALORACIÓN DE LOS ACTIVOS Y PASIVOS POR IMPUESTO CORRIENTE Y DIFERIDO}

Los activos y pasivos por impuesto corriente se valorarán por las cantidades que se espera recuperar o pagar, respectivamente, de las autoridades fiscales, de acuerdo con la normativa vigente o aprobada y pendiente de publicación en la fecha de cierre del ejercicio.

Por su parte, los activos y pasivos por impuesto diferido se valorarán de acuerdo con los tipos de gravamen esperados en el momento de su reversión. Para ello, se tendrán en cuenta dos consideraciones:

a) La normativa vigente o aprobada y pendiente de publicación al cierre del ejercicio, y

b) La forma en la que se espera recuperar o pagar el activo o el pasivo.

Esta última cuestión resulta coherente con lo previsto en el párrafo 51 de la NIC 12, según el cual, la valoración de activos y pasivos por impuestos diferidos debe reflejar las consecuencias fiscales que se derivarían de la forma en que la empresa espera, a la fecha del balance, recuperar el importe en libros de sus activos o liquidar el importe en libros de sus pasivos.

Sucede, en efecto, que en algunos casos, la forma en la que se espere recuperar o liquidar el importe en libros de un activo o un pasivo, respectivamente, afecta a una $o$ a las dos circunstancias siguientes:

a) El tipo aplicable cuando se recupere el importe en libros de activo o se liquide el pasivo.

b) La base fiscal del activo o el pasivo.

En esos casos, la valoración de los activos y pasivos por impuesto diferido se efectuará utilizando el tipo y la base fiscal que 
procedan en función de la forma en que se espera recuperar o pagar la partida correspondiente.

\section{Ejemplo n ${ }^{0}$ 13.-}

Supongamos un activo con un valor contable de 500 u.m. y una base fiscal de 300 u.m. En caso de venta, a las ganancias se les aplicaría un tipo impositivo del $20 \%$, al resto de rendimientos se les aplica un tipo del $30 \%$.

Solución.-

La diferencia temporaria que existe en este caso es de 200 u.m. Se trata de una diferencia temporaria imponible, que genera un pasivo por impuestos diferidos, cuyo importe debemos determinar.

El pasivo derivado de la diferencia temporaria depende de si la empresa recupera el valor en libros del activo (500) mediante la venta, o mediante el uso.

En el primer caso, es decir, cuando la empresa piensa proceder a la venta, reconocerá un pasivo que se corresponderá con el 30\% de 200, es decir, 60 u.m. En el segundo caso, si prevé conservar el activo y recuperar su valor mediante el uso, el pasivo sería del $20 \%$ de 200, ó sea, 40 u.m.

Evidentemente, el importe por el que inicialmente se valoran activos y pasivos por impuestos diferidos puede variar posteriormente como consecuencia de modificaciones en la legislación tributaria, entre las que se incluyen los cambios en los tipos de gravamen, y también, debido a la evolución de la situación económica de la empresa. Dichos ajustes de valor se considerarán como gasto o ingreso, según corresponda, por impuesto diferido, que se imputará a la cuenta de pérdidas y ganancias, salvo que se relacione con partidas que previamente se cargaron o abonaron al patrimonio neto, en cuyo caso, se abonarán directamente en éste.

En el caso particular de combinaciones de negocio, en las que inicialmente no se reconocieron activos por impuestos diferidos de la empresa adquirida, por no cumplirse en ese momento los criterios de reconocimiento, y posteriormente proceda dicho reconocimiento, además de los ajustes indicados en el párrafo anterior, deberá reducirse el fondo de comercio hasta el importe por el que hubiera sido contabilizado, de haberse reconocido en la fecha de adquisición los mencionados activos por impuestos diferidos. La reducción del fondo de comercio se registrará como un gasto en la cuenta de pérdidas y ganancias. 
Ejemplo n ${ }^{0}$ 14.-

Supongamos que la empresa A adquirió el 1-6-08 la totalidad del patrimonio de la empresa $B$, surgiendo en la operación un fondo de comercio de 5.000 u.m. La entidad adquirida tenía en dicha fecha, diferencias temporarias deducibles por importe de de 1.200 u.m., siendo el tipo impositivo del 35\%. En ese momento no pudo reconocerse el activo correspondiente a las mencionadas diferencias temporarias.

A 31-12-09 la situación económica de la empresa ha mejorado notablemente, estimándose ahora, que podrán obtenerse ganancias fiscales futuras en cuantía suficiente para poder registrar el activo no reconocido en su momento. El tipo impositivo ha pasado a ser del $30 \%$.

\section{Solución.-}

En primer lugar, reconoceríamos el activo no registrado en su momento valorándolo de acuerdo con el tipo de gravamen esperado en el momento de la reversión, esto es, el 30\%:

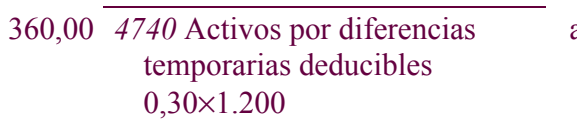
638 Ajustes positivos en la
imposición sobre beneficios
360,00

En segundo lugar, el fondo de comercio deberá reducirse hasta el importe por el que hubiera sido contabilizado de haberse reconocido en la fecha de adquisición el anterior activo fiscal. Si tal reconocimiento hubiera tenido lugar, el mencionado activo, se habría valorado de acuerdo con el tipo vigente en ese momento, el $35 \%$, por lo que su valor se situaría en el 35\% de 1.200 u.m., es decir, 420 u.m. En ese importe debemos reducir el Fondo de comercio:

\begin{tabular}{cc}
$\begin{array}{c}670 \text { Pérdidas procedentes del } \\
\text { inmovilizado intangible }\end{array}$ \\
\hline
\end{tabular}

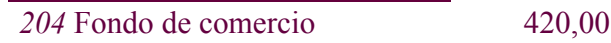

Por último, a efectos de la valoración de los activos y pasivos por impuesto diferido, es necesario apuntar que, tanto el PGC como la NIC 12, establecen que los mismos no deben ser descontados. Evaluar de forma fiable los activos y pasivos por impuestos diferidos exigiría, de acuerdo con la NIC 12, determinar la distribución en el tiempo de cada diferencia temporaria, lo cual, en la mayoría de los casos, resulta impracticable o muy complejo. Por esa razón, la NIC 12 desestima exigir el descuento. Si aún así, este estuviera permitido, daría lugar a unas cifras de activos y pasivos por impuestos diferidos que no resultarían comparables entre empresas. Por todo ello, la mencionada NIC ni exige ni permite el descuento de estos saldos. 


\section{GASTO (INGRESO) POR IMPUESTO SOBRE BENEFICIOS}

El gasto (ingreso) por impuesto sobre beneficios se obtiene de la suma de los dos conceptos siguientes:

A) Impuesto corriente: Concepto fiscal que recoge la cantidad a pagar o a recuperar como consecuencia de la liquidación fiscal del ejercicio.

A efectos de calcular el importe correspondiente al impuesto corriente del ejercicio, es necesario tener en cuenta que el mismo, se verá disminuido como consecuencia de las deducciones y otras ventajas fiscales en la cuota del impuesto, excluidas las retenciones y pagos a cuenta, así como por las pérdidas fiscales procedentes de ejercicios anteriores que se compensan en el actual.

El impuesto corriente se reconocerá como un pasivo en la medida que esté pendiente de pago. Por el contrario, cuando la cantidad ya pagada exceda del impuesto corriente, el exceso deberá reconocerse como un activo.

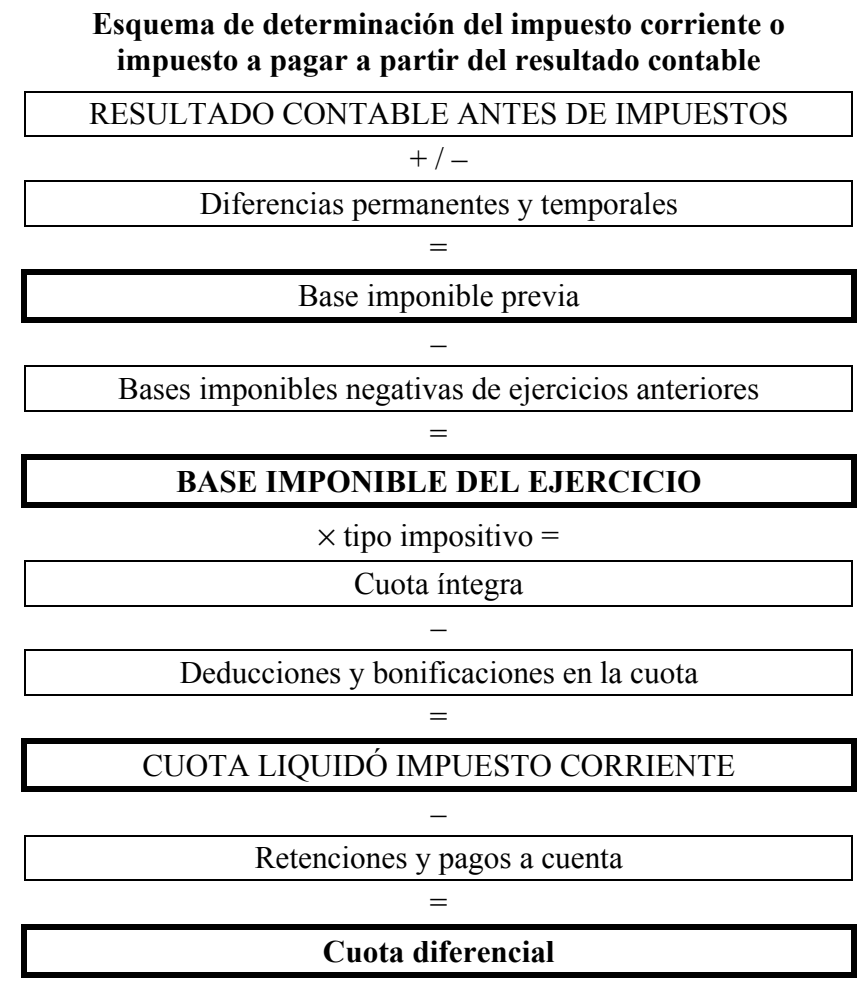


B) Impuesto diferido: concepto contable que se corresponde con el efecto impositivo que se produce por el diferente tratamiento contable y fiscal de los hechos económicos. La aparición o extinción de pasivos/activos por impuesto diferido, cuyo origen se ha analizado con anterioridad, da lugar al gasto/ingreso por impuesto diferido.

Con respecto al reconocimiento de los impuestos corrientes y diferidos, la NIC 12 establece el principio general de que la contabilización de los efectos fiscales de una determinada transacción debe ser coherente con el registro contable que se establezca para la misma. Asumiendo dicho principio, el PGC establece que los impuestos corrientes y diferidos deben imputarse a la Cuenta de Pérdidas y Ganancias salvo en los siguientes casos:

- Si se relacionan con una transacción que se hubiese reconocido directamente en el patrimonio neto: en estos casos los impuestos corrientes o diferidos se cargarán o abonarán a cuentas de patrimonio neto.

- Si surgen en una combinación de negocios: el cargo o abono correspondiente se efectuaría a la cuenta representativa del fondo de comercio.

\section{REGISTRO CONTABLE DEL IMPUESTO SOBRE BENEFICIOS}

Teniendo en cuenta todos los conceptos desarrollados con anterioridad, incluimos a continuación, una serie de ejemplos relativos al registro del impuesto sobre beneficios en el PGC. Para ello, comenzamos con la exposición de las cuentas aplicables a tal fin.

a) Cuentas relativas a activos y pasivos corrientes:

4709. Hacienda Pública deudora por devolución de impuestos

473. Hacienda Pública, retenciones y pagos a cuenta

4752. Hacienda Pública acreedora por impuesto de sociedades

b) Cuentas relativas a activos y pasivos por impuestos diferidos:

4740. Activos por diferencias temporarias deducibles

4742. Derechos por deducciones y bonificaciones pendientes de aplicar 4745. Crédito por pérdidas a compensar del ejercicio 
c) Cuentas relativas a gastos incluidos en Pérdidas y Ganancias:

6300. Impuesto corriente

6301. Impuesto diferido

633. Ajustes negativos en la imposición sobre beneficios

638. Ajustes positivos en la imposición sobre beneficios

d) Cuentas relativas a gastos imputados al patrimonio neto:

8300. Impuesto corriente

8301. Impuesto diferido

833. Ajustes negativos en la imposición sobre beneficios

838. Ajustes positivos en la imposición sobre beneficios

\section{Ejemplo $\mathbf{n}^{\mathbf{0}}$ 15.- Diferencias temporarias imponibles}

La sociedad QUINCE ha obtenido en el ejercicio 2007 un resultado contable antes de impuestos de 50.000 u.m.

Al inicio del ejercicio adquirió una máquina por 25.000 u.m, que contablemente se amortiza en 5 años de forma lineal.

A efectos fiscales, dicha máquina goza de libertad de amortización y la sociedad decide amortizarla en su totalidad en el ejercicio 2007.

No hay ninguna otra diferencia entre contabilidad y fiscalidad.

Tipo de gravamen: 30\%. Total retenciones y pagos a cuenta: 5.000

SE PIDE: Contabilizar el impuesto de sociedades correspondiente al año 2007.

\section{Solución.-}

Compararemos para el activo:

\begin{tabular}{|c|c|c|c|c|c|c|}
\hline Año & $\begin{array}{c}\text { Valor } \\
\text { contable }\end{array}$ & $\begin{array}{c}\text { Base } \\
\text { fiscal }\end{array}$ & $\begin{array}{c}\text { Diferencia } \\
\text { temporaria } \\
\text { imponible total }\end{array}$ & $\begin{array}{c}\text { Dif. temp. } \\
\text { del ejercicio } \\
\text { DTf - DTi }\end{array}$ & $\begin{array}{c}\text { Pasivo por } \\
\text { DT } \\
\text { imponible }\end{array}$ & $\begin{array}{c}\text { Variación } \\
\text { Pasivo por } \\
\text { DT imponible }\end{array}$ \\
\hline 2007 & 20.000 & 0 & 20.000 & 20.000 & 6.000 & +6.000 \\
\hline 2008 & 15.000 & 0 & 15.000 & -5.000 & 4.500 & -1.500 \\
\hline 2009 & 10.000 & 0 & 10.000 & -5.000 & 3.000 & -1.500 \\
\hline 2010 & 5.000 & 0 & 5.000 & -5.000 & 1.500 & -1.500 \\
\hline 2011 & 0 & 0 & 0 & & 0 & -1.500 \\
\hline
\end{tabular}

Calculamos la diferencia temporal para cada ejercicio: 


\begin{tabular}{|c|c|c|c|}
\hline Año & $\begin{array}{c}\text { Gasto } \\
\text { contable }\end{array}$ & $\begin{array}{c}\text { Gasto } \\
\text { fiscal }\end{array}$ & $\begin{array}{c}\text { Diferencia } \\
\text { temporal }\end{array}$ \\
\hline 2007 & 5.000 & 25.000 & -20.000 \\
\hline 2008 & 5.000 & - & +5.000 (rev.) \\
\hline 2009 & 5.000 & - & +5.000 (rev.) \\
\hline 2010 & 5.000 & - & +5.000 (rev.) \\
\hline 2011 & 5.000 & - & +5.000 (rev.) \\
\hline Total & $\mathbf{2 5 . 0 0 0}$ & $\mathbf{2 5 . 0 0 0}$ & $\mathbf{0}$ \\
\hline
\end{tabular}

Se cumplimenta la declaración a Hacienda:

\begin{tabular}{|c|c|}
\hline de impuestos & 50.000 \\
\hline Diferencia temporal negativa .. & $(20.000)$ \\
\hline 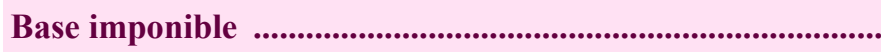 & 30.000 \\
\hline Tipo de gravamen & $30 \%$ \\
\hline 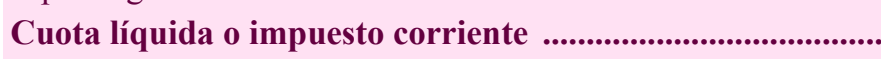 & 9.000 \\
\hline enta & $(5.000)$ \\
\hline Hota difarancial & 4.000 \\
\hline
\end{tabular}

Se recoge, en primer lugar y con cargo a resultados, el pasivo por impuesto corriente:

9.000,00 6300 Impuesto corriente

a 4752 H.P. acreedora por impuesto sobre sociedades

a 473 H.P. retenciones y pagos a cuenta

El impuesto corriente, 9.000, es la cantidad que Hacienda exige legalmente a la sociedad, independientemente de que exista, o no, un gasto/ingreso por impuesto diferido.

En segundo lugar, hay que reconocer el impuesto diferido. Existe una diferencia temporaria imponible, que da lugar a un pasivo por diferencias temporarias imponibles, ya que en el futuro habrá un mayor pago de impuestos:

$6.000,0 0 \longdiv { 6 3 0 1 \text { Impuesto diferido a } }$

\begin{tabular}{l}
479 Pasivos por diferencias \\
temporarias imponibles \\
\hline
\end{tabular}

$6.000,00$

El gasto total por el impuesto es: $9.000+6.000=15.000$, es decir, superior al impuesto a pagar, esa diferencia se pagará en ejercicios siguientes.

\section{Ejemplo $\mathbf{n}^{0}$ 16.- Reversión de diferencias temporarias imponibles}

En el ejercicio siguiente, la misma sociedad ha obtenido un resultado antes de impuestos de 30.000 u.m.

Tipo de gravamen: $30 \%$

Deducciones aplicadas en el ejercicio: 3.000 u.m.

Retenciones y pagos a cuenta: 4.000

SE PIDE: Contabilizar el impuesto de sociedades correspondiente al año 2008. 


\section{Solución.-}

Se cumplimenta la declaración a Hacienda:

\begin{tabular}{|c|c|}
\hline Resultado contable antes de impuestos & 30.000 \\
\hline Diferencia temporal positiva (reversión) & +5.000 \\
\hline 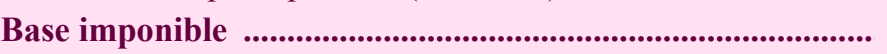 & 35.000 \\
\hline Tipo de gravamen & $30 \%$ \\
\hline Cuota íntegra & 10.500 \\
\hline Deducciones y bonificaciones en la cuota ... & $(3.000)$ \\
\hline Cuota líquida 0 impuesto corriente .............. & 7.500 \\
\hline Retenciones y pagos a cuenta ..... & $(4.000)$ \\
\hline Cuota diferencial ......................... & 3.500 \\
\hline
\end{tabular}

Se recoge, en primer lugar y con cargo a resultados, el pasivo por impuesto corriente:
$7.500,00 \quad 6300$ Impuesto corriente
a 4752 H.P. acreedora por impuesto sobre sociedades
a 473 H.P. retenciones y pagos a cuenta

El impuesto corriente, 7.500, es la cantidad que Hacienda exige legalmente a la sociedad, independientemente de que exista, o no, un gasto/ingreso por impuesto diferido.

En segundo lugar, hay que reconocer la reversión del impuesto diferido: revierte, en este caso, una diferencia temporaria imponible, lo que supone un mayor pago de impuestos en el ejercicio:
$1.500,0 0 \longdiv { 4 7 9 \text { Pasivos por diferencias } }$ temporarias imponibles

6301 Impuesto diferido $\quad 1.500,00$

El gasto total por el impuesto es: $7.500-1.500=5.000$, es decir, inferior al impuesto a pagar, pues en este ejercicio se paga ya una parte del pasivo por impuestos diferidos que surgió el ejercicio anterior.

\section{Ejemplo n ${ }^{0}$ 17.- Aparición y compensación de bases imponibles negativas}

Una empresa obtiene en el ejercicio, como consecuencia de un hecho aislado, un resultado contable antes de impuestos negativo de 100.000 u.m.

No existen diferencias entre contabilidad y fiscalidad y las retenciones y pagos a cuenta ascienden a 10.000 u.m.

En el ejercicio siguiente, la misma empresa obtiene un resultado contable antes de impuestos de 120.000, por lo que decide compensar las pérdidas anteriores.

Las retenciones y pagos a cuenta han ascendido este ejercicio a 5.000 u.m.

SE PIDE: Contabilizar el impuesto de sociedades correspondiente a los dos ejercicios.

\section{Solución.-}

Se cumplimenta la declaración a Hacienda: 


\section{a) Año 1}

Liquidación del impuesto:

\begin{tabular}{|c|c|}
\hline Resultado contable antes de impuestos & 100.000 \\
\hline Base imponible & 100.000 \\
\hline gravamen & $30 \%$ \\
\hline
\end{tabular}

No existe, en este caso, gasto o ingreso por impuesto corriente, no existen cantidades ni a pagar ni a recuperar (de acuerdo con nuestra legislación) de la Hacienda Pública derivadas de la liquidación fiscal del ejercicio.

Contabilización del crédito impositivo:

$$
\begin{aligned}
& \text { 30.000,00 } 4745 \text { Crédito por pérdidas a } \\
& \text { compensar }(0,30 \times 100.000) \\
& \text { a } \overline{6301 \text { Impuesto diferido }} \quad 30.000,00
\end{aligned}
$$

Por el derecho a la devolución directa de lo ya abonado a cuenta del impuesto durante el ejercicio, registramos:

$\begin{array}{lllll}10.000,00 & \begin{array}{c}4709 \text { H.P. deudora por } \\ \text { devolución de impuestos }\end{array} & \begin{array}{l}\text { a } \\ \text { deventa }\end{array} & 10.000,00\end{array}$

El ingreso total por el impuesto asciende a 30.000 u.m. y se corresponde con el saldo acreedor de la cuenta 6301.

\begin{tabular}{|c|c|}
\hline Resultado contable antes de impuestos & 120.000 \\
\hline Base imponible previa ......... & 120.000 \\
\hline Compensación base imponible ejercicios anteriores & 100.000 \\
\hline Base imponible del ejercicio & 20.000 \\
\hline Tipo de gravamen & $30 \%$ \\
\hline Cuota líquida o impuesto corriente ........ & 6.000 \\
\hline Retenciones y pagos a cuenta & 5.000 \\
\hline Cuota diferencial ...................... & 1.00 \\
\hline
\end{tabular}

\section{b) Año 2}

Liquidación del impuesto:

Contabilización del impuesto a pagar:

$6.000,006300$ Impuesto corriente

a 4752 H.P. acreedora por impuesto sobre sociedades

a 473 H.P. retenciones y pagos a cuenta

Por la cancelación del crédito, que supone un menor pago de impuestos en el ejercicio:

$30.000,0 0 \longdiv { 6 3 0 1 \text { Impuesto diferido a } } \begin{array} { c } { 4 7 4 5 \text { Crédito por pérdidas a } } \\ { \text { compensar } } \end{array}$

Gasto por impuesto del ejercicio: $6.000+30.000=36.000$. 


\section{Ejemplo $\mathbf{n}^{0}$ 18.- Revalorización de activos financieros disponibles para la venta}

La empresa SEIS obtiene en el ejercicio 2008 un resultado contable antes de impuestos de 90.000 u.m. Al cierre del ejercicio ha contabilizado la revalorización en 10.000 u.m. de un activo financiero disponible para la venta inicialmente se habia valorado en 20.000 u.m.

Tipo de gravamen: $30 \%$

Las retenciones y pagos a cuenta ascienden a 10.000 u.m.

SE PIDE: Contabilizar el impuesto de sociedades correspondiente al año 2008.

\section{Solución.-}

En este caso, la ganancia por la revalorización no pasará por la cuenta de resultados sino que afectará al patrimonio neto, por lo que no tendrá que tributar hasta que se vendan los títulos. Surgirá una diferencia temporaria:

\begin{tabular}{|c|c|c|c|c|c|c|}
\hline Año & $\begin{array}{c}\text { Valor } \\
\text { contable }\end{array}$ & $\begin{array}{c}\text { Base } \\
\text { fiscal }\end{array}$ & $\begin{array}{c}\text { Diferencia } \\
\text { temporaria } \\
\text { imponible total }\end{array}$ & $\begin{array}{c}\text { Dif. temp. } \\
\text { del ejercicio } \\
\text { DTf - DTi }\end{array}$ & $\begin{array}{c}\text { Pasivo por } \\
\text { DT } \\
\text { imponible }\end{array}$ & $\begin{array}{c}\text { Variación } \\
\text { Pasivo por } \\
\text { DT imponible }\end{array}$ \\
\hline 2007 & 30.000 & 20.000 & 10.000 & 10.000 & 3.000 & +3.000 \\
\hline
\end{tabular}

Se trata de una diferencia temporaria imponible, que implica un mayor importe a pagar en el ejercicio en el que se recupere el valor contable del activo.

No es una diferencia temporal, la revalorización no forma parte ni del resultado contable ni de la base imponible.

Por la revalorización del activo contabilizaríamos:

$\begin{array}{llll}10.000,00 & \begin{array}{l}540 \text { Inversiones financieras a c/p a } \\ \text { en I.P. }\end{array} & \begin{array}{l}900 \text { Beneficios en activos financieros } \\ \text { disponibles para la venta }\end{array} & 10.000,00\end{array}$

Por el efecto impositivo de la diferencia temporaria:

$\begin{array}{llll}3.000,00 & \begin{array}{l}8301 \text { Impuesto diferido } \\ 0,30 \times 10.000\end{array} & \text { a } & \begin{array}{l}479 \text { Pasivos por diferencias } \\ \text { temporarias imponibles }\end{array} \\ & 3.000,00\end{array}$

Por la imputación al Patrimonio Neto del importe de la revalorización, neto del efecto impositivo:

\begin{tabular}{|c|c|c|}
\hline \multirow[t]{2}{*}{$10.000,00$} & \multirow[t]{2}{*}{$\begin{array}{l}900 \text { Beneficios en activos financieros } \\
\text { disponibles para la venta }\end{array}$} & $\begin{array}{l}\text { a } 133 \text { Ajustes por valoración de activos } \\
\text { financieros disponibles para la } \\
\text { venta }\end{array}$ \\
\hline & & a 8301 Impuesto diferido \\
\hline
\end{tabular}

El impuesto a pagar quedaría así:

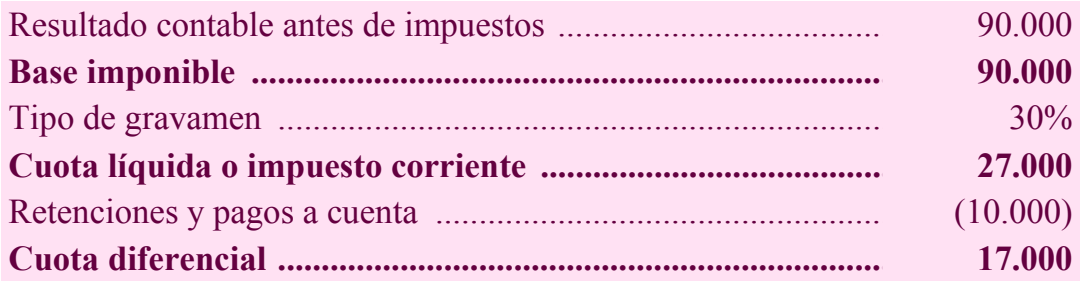


Contabilización:

\begin{tabular}{ccc}
\cline { 2 - 3 } 6300 Impuesto corriente & a $\begin{array}{c}4752 \text { H.P. acreedora por } \\
\text { impuesto sobre sociedades }\end{array}$ \\
& a $\begin{array}{c}473 \text { H.P. retenciones y pagos a } \\
\text { cuenta }\end{array}$ \\
\hline
\end{tabular}
cuenta $10.000,00$

El gasto devengado por el impuesto asciende a 27.000. Además, se han imputado gastos por impuestos diferidos al patrimonio neto por importe de 3.000 .

\section{Ejemplo $n^{0}$ 19.- Venta de activos financieros disponibles para la venta, previamente revalorizados}

La empresa del ejercicio anterior obtiene en el ejercicio siguiente un resultado contable antes de impuestos de 100.000 u.m., habiendo vendido durante el ejercicio los títulos anteriormente mencionados por un importe de 35.000 u.m.

Tipo de gravamen: $30 \%$

Las retenciones y pagos a cuenta ascienden a 5.000 u.m.

SE PIDE: Contabilizar el impuesto de sociedades correspondiente al ejercicio.

\section{Solución.-}

La empresa registrará la venta de los títulos de la siguiente forma:

\begin{tabular}{|c|c|c|c|c|}
\hline & & & & \\
\hline $35.000,00$ & 57 Tesorería & $\mathrm{a}$ & $\begin{array}{l}540 \text { Inversiones financieras a c/p } \\
\text { en I.P. }\end{array}$ & $30.000,00$ \\
\hline & & $\mathrm{a}$ & $\begin{array}{l}766 \text { Beneficios en participaciones } \\
\text { y valores representativos de } \\
\text { deuda }\end{array}$ & $5.000,00$ \\
\hline
\end{tabular}

Por la transferencia a resultados del importe positivo acumulado en el patrimonio neto:

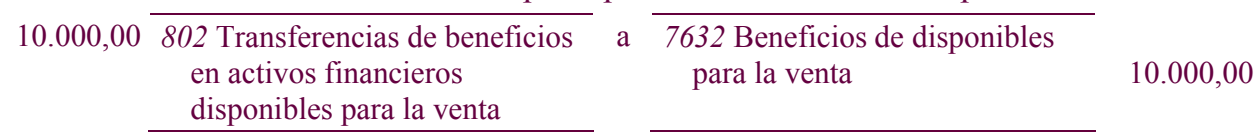

No existe diferencia temporal, el resultado positivo de la operación, 15.000, forma parte tanto del resultado contable como de la base imponible.

El impuesto a pagar quedaría así:

\begin{tabular}{|c|c|}
\hline Resultado contable antes de impuestos & 100.000 \\
\hline Base imponible & 100.000 \\
\hline Tipo de gravamen ....................... & $30 \%$ \\
\hline Cuota líquida o impuesto corriente .......................................... & 30.000 \\
\hline Retenciones y pagos a cuenta & $(5.000)$ \\
\hline Cuota diferencial ......................... & 25.000 \\
\hline
\end{tabular}


$30.000,00 \quad 6300$ Impuesto corriente a 4752 H.P. acreedora por impuesto sobre sociedades

a 473 H.P. retenciones y pagos a cuenta
$25.000,00$

$5.000,00$

Revierte el efecto impositivo:

\begin{tabular}{|c|c|c|c|c|c|c|}
\hline Año & $\begin{array}{c}\text { Valor } \\
\text { contable }\end{array}$ & $\begin{array}{c}\text { Base } \\
\text { fiscal }\end{array}$ & $\begin{array}{c}\text { Diferencia } \\
\text { temporaria } \\
\text { imponible total }\end{array}$ & $\begin{array}{c}\text { Dif. temp. } \\
\text { del ejercicio } \\
\text { DTf - DTi }\end{array}$ & $\begin{array}{c}\text { Pasivo por } \\
\text { DT } \\
\text { imponible }\end{array}$ & $\begin{array}{c}\text { Variación } \\
\text { Pasivo por } \\
\text { DT imponible }\end{array}$ \\
\hline 2008 & 30.000 & 20.000 & 10.000 & 10.000 & 3.000 & +3.000 \\
\hline 2009 & 0 & 0 & 0 & -10.000 & 0 & -3.000 \\
\hline
\end{tabular}

$3.000,00479$ Pasivos por diferencias

a 8301 Impuesto diferido

$3.000,00$ temporarias imponibles

El gasto por impuesto asciende a 30.000 u.m., habiéndose imputado al patrimonio neto un ingreso de 3.000 u.m.

Al cierre del ejercicio se regularizarían las cuentas de los grupos 8 y 9 así:

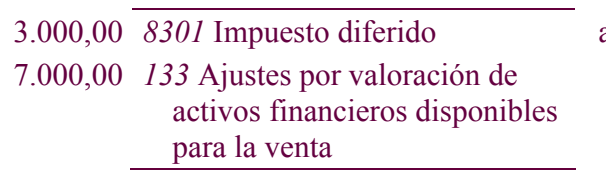

a 802 Transferencia de beneficios

en activos financieros

disponibles para la venta

$10.000,00$

\section{Ejemplo $\mathbf{n}^{\circ}$ 20.- Efecto impositivo de las subvenciones de capital}

La sociedad CONTASA recibe, a comienzos de 200x, una subvención para financiar la compra de maquinaria diversa por un importe de 150.000€. A la maquinaria se le estima una vida útil de 5 años, sin valor residual.

Resultado contable antes de impuestos: 800.000€.

Retenciones y pagos a cuenta: $175.000 €$

Tipo impositivo: $30 \%$.

\section{Solución.-}

Calculamos la diferencia temporaria que origina la subvención:

Base fiscal de activo financiado por la subvención: $150.000-150.000=0$. Fiscalmente, la subvención se integra en la base imponible a medida que se amortiza el activo subvencionado, por lo tanto, en este caso, la base fiscal del citado activo, lo "deducible fiscalmente en el futuro" es 0 , las cuotas de amortización se compensan con los traspasos a resultados de la subvención.

Valor contable del activo: 150.000 .

Diferencia temporaria: 150.000 . Se trata de una diferencia temporaria imponible, que genera un pasivo de 45.000 (30\% de 150.000). Este tipo de diferencias se corresponde con las contempladas en el apartado 2.1.b primero de la norma de 


\begin{tabular}{|c|c|}
\hline Resultado contable antes de impuestos & 800.000 \\
\hline Diferencias temporales & - \\
\hline 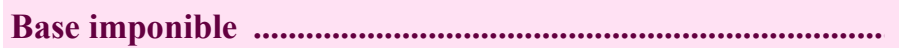 & 800.000 \\
\hline Tipo de gravamen & $30 \%$ \\
\hline Cuota líquida o impuesto corriente ....... & 240.000 \\
\hline Retenciones y pagos a cuenta & 175.000 \\
\hline Cuota diferencial .................... & 65.000 \\
\hline
\end{tabular}

No existen diferencias temporales entre el resultado contable y la base imponible; la amortización y el traspaso de la subvención al resultado del ejercicio forman parte de ambas magnitudes.

\section{Ejemplo $\mathbf{n}^{\mathbf{0}}$ 21.- Efecto impositivo de las donaciones}

Al final del ejercicio 200x la sociedad ANSA recibe una donación consistente en mobiliario diverso cuyo valor razonable, coincidente con el valor de mercado, asciende a 400.000€. La amortización del mismo, se realizará durante los 5 años siguientes de forma lineal.

El resultado contable antes de impuestos asciende a 500.000€ y las retenciones y pagos a cuenta a 80.000€.

\section{Solución.-}

Comprobamos, en primer lugar, si existe o no diferencia temporaria:

Base fiscal del activo donado: 400.000€. Fiscalmente, el valor de mercado de las transmisiones lucrativas (400.000€, en este caso), se integra en la base imponible del ejercicio en el que se produce dicha transmisión, por lo que en ejercicios siguientes resultarán fiscalmente deducibles en su totalidad las cuotas de amortización de dichos bienes.

Valor contable del activo: $400.000 €$

Diferencia temporaria: 0

Registro contable de la donación:

\begin{tabular}{|c|c|c|c|}
\hline $400.000,00$ & 216 Mobiliario & $\mathrm{a}$ & $\begin{array}{l}941 \text { Ingresos por donaciones y } \\
\text { legados de capital }\end{array}$ \\
\hline
\end{tabular}

La donación tributa en este ejercicio, razón por la cual, debe reconocerse la deuda con Hacienda Pública, con cargo a la cuenta (8300) Impuesto corriente, puesto que la operación correspondiente no ha afectado al resultado del ejercicio, sino al patrimonio neto:

\begin{tabular}{|c|c|c|}
\hline $120.000,00$ & $\begin{array}{l}8300 \text { Impuesto corriente } \\
0,30 \times 400.000\end{array}$ & $\begin{array}{l}4752 \text { Hacienda Pública, acreedora } \\
\text { por impuesto sobre sociedades }\end{array}$ \\
\hline
\end{tabular}

Al cierre, regularizamos las cuentas de los grupos 8 y 9 contra la correspondiente cuenta de patrimonio neto: 


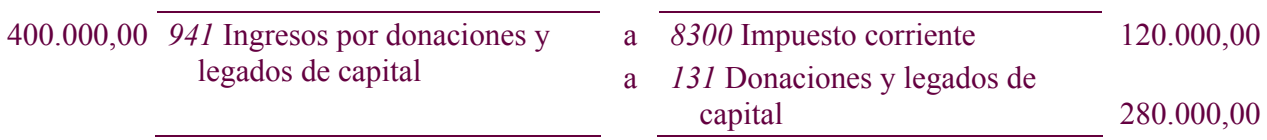

En este momento, la donación figura en el patrimonio neto por su importe neto del efecto impositivo.

El impuesto a pagar quedaría así:

\begin{tabular}{|c|c|}
\hline Resultado contable antes de impuestos .... & 500.000 \\
\hline Diferencias temporales . & - \\
\hline Base imponible ................. & 500.000 \\
\hline Tipo de gravamen & $30 \%$ \\
\hline Cuota líquida 0 impuesto corriente ....................................... & 150.000 \\
\hline Retenciones y pagos a cuenta ............. & $(80.000)$ \\
\hline 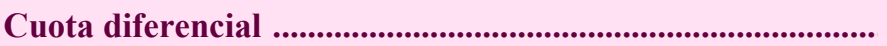 & 70.000 \\
\hline
\end{tabular}

No se considera la existencia de diferencias entre el resultado contable y la base imponible, pues el efecto del aumento en esta última como consecuencia de la donación ya se ha contabilizado con cargo el patrimonio neto, al no haber afectado al resultado del ejercicio:

\begin{tabular}{|c|c|c|c|c|}
\hline \multirow{3}{*}{$150.000,00$} & \multirow{3}{*}{6300 Impuesto corriente } & \multirow[b]{2}{*}{$\mathrm{a}$} & & \\
\hline & & & $\begin{array}{l}4752 \text { Hacienda Pública, acreedora } \\
\text { por impuesto sobre sociedades }\end{array}$ & $70.000,00$ \\
\hline & & $\mathrm{a}$ & $\begin{array}{l}473 \text { H.P. retenciones y pagos a } \\
\text { cuenta }\end{array}$ & $80.000,00$ \\
\hline
\end{tabular}

Por la amortización y traspaso a resultados de la parte proporcional de la donación a finales del año siguiente:

\begin{tabular}{|c|c|c|c|}
\hline & \multirow[b]{2}{*}{$\mathrm{a}$} & \\
\hline $80.000,00$ & $\begin{array}{l}681 \text { Amortización del inmovilizado } \\
\text { material }(400.000 / 5)\end{array}$ & & $\begin{array}{l}281 \text { Amortización acumulada del } \\
\text { inmovilizado material }\end{array}$ \\
\hline $80.000,00$ & $\begin{array}{l}841 \text { Transferencia de donaciones } \\
\text { y legados de capital }(400.000 / 5)\end{array}$ & $\mathrm{a}$ & $\begin{array}{l}746 \text { Subvenciones, donaciones y } \\
\text { legados de capital transferidos } \\
\text { al resultado del ejercicio }\end{array}$ \\
\hline
\end{tabular}

Fiscalmente, en cada uno de los ejercicios en los que la donación se traspasa a resultados, habrá que realizar un ajuste negativo al resultado contable a efectos de calcular la base imponible del impuesto, pues dicho ingreso ya tributó en el ejercicio en el que la donación tuvo lugar. De acuerdo con el movimiento previsto para la cuenta "8301. Impuesto diferido", por el efecto impositivo derivado de la transferencia a resultados de ingresos imputados directamente al patrimonio neto que hubieran ocasionado el correspondiente impuesto corriente en ejercicios previos, la empresa abonará la mencionada cuenta con cargo a la cuenta "6301. Impuesto diferido". En este caso, el registro contable sería:

\begin{tabular}{cccc}
\cline { 2 - 3 } $24.000,00$ & $\begin{array}{c}6301 \text { Impuesto diferido } \\
0,30 \times 80.000\end{array}$ & a & 24.000,00
\end{tabular}

Por la regularización al cierre de las cuentas de los grupos 8 y 9 :

\begin{tabular}{cl}
$24.000,00$ & 8301 Impuesto diferido \\
\cline { 2 - 2 } & $\begin{array}{c}131 \text { Donaciones y legados de } \\
\text { capital }\end{array}$ \\
\hline
\end{tabular}

841 Transferencia de donaciones

y legados de capital $\quad 80.000,00$ 
El saldo en estos momentos de la cuenta "131. Donaciones y legados de capital", asciende a 224.000, importe que se corresponde con el valor de la donación, neto del efecto impositivo, pendiente de imputar a resultados.

Si suponemos ahora que el resultado contable antes de impuestos del ejercicio ha sido de $600.000 €$, habiendo ascendido las retenciones y pagos a cuenta a $90.000 €$, la liquidación del impuesto sería la siguiente:

\begin{tabular}{|c|c|}
\hline esultado contable antes de & 600.000 \\
\hline Ajuste negativo & $(80.000)$ \\
\hline Base imponible ........... & 520.000 \\
\hline Tipo de gravamen & $30 \%$ \\
\hline Cuota líquida o impuesto corriente ... & 156.000 \\
\hline Retenciones y pagos a cuenta & $(90.000)$ \\
\hline Cuota diferencial ....... & 66.000 \\
\hline
\end{tabular}

El ajuste negativo se corresponde con el hecho de que el ingreso contable derivado del traspaso de la donación al resultado del ejercicio no se incorpora a la base imponible del ejercicio, pues ya tributó en su momento:

\begin{tabular}{llll}
\cline { 2 - 3 } 156.000,00 & a Impuesto corriente & $\begin{array}{c}4752 \text { Hacienda Pública, acreedora } \\
\text { por impuesto sobre sociedades }\end{array}$ & $66.000,00$ \\
& a $\begin{array}{l}473 \text { H.P. retenciones y pagos a } \\
\text { cuenta }\end{array}$ & $90.000,00$
\end{tabular}

\section{BIBLIOGRAFÍA}

Amador, S. y I. Carazo (2008) Plan General de Contabilidad. Comentarios y casos prácticos, $9^{a}$ edición. Madrid: CEF.

CASO FERNÁNDEZ, J.C. de (2008) "El Impuesto sobre beneficios en el NPGC (I)", Partida Doble, $\mathrm{n}^{\circ}$ 195, enero, pp. 14-32.

- (2008) "El Impuesto sobre beneficios en el NPGC (II)", Partida Doble, $\mathrm{n}^{\circ}$ 196, febrero, pp. 10-21.

Corona Romero, e.; J. Talavero Sanguino, F. García martínez, Á. Monzón SÁNCHEZ, R. LóPEZ GALINDO, J. CLAVO GONZÁlEZ-VALLINAS y J. VARELA CRUZEIRO (2005) Aplicación de las normas internacionales de contabilidad adoptadas por la Unión Europea (NICes). Valencia: CISS.

DOSSIER PRÁCTICO FRANCIS LEFEBVRE (2008) Sistemática Memento. Plan General Contable. 125 Supuestos prácticos. Madrid: Ediciones Francis Lefebvre. 
García-Olmedo Domínguez, R. y E. Corona Romero (2007) "La contabilización del Impuesto sobre Sociedades según el Borrador del PGC" (I), Técnica Contable, $\mathrm{n}^{\circ} 702$, noviembre, pp. 24-37.

- y - (2007) "La contabilización del Impuesto sobre Sociedades según el Borrador del PGC" (II), Técnica Contable, n 703, diciembre, pp. 44-49.

INTERNATIONAL ACCOUNTING STANDARDS BOARD (2006) Normas Internacionales de información financiera (NIIF), texto completo de las Normas Internacionales de Información Financiera emitidas a 1 de enero de 2006, traducción al español publicada por CISS-PRAXIS con la autorización de la IASCF.

KPMG INTERNATIONAL FINANCIAL REPORTING GROUP (2006) Las NIIF comentadas. Guía práctica de KPMG para comprender las Normas Internacionales de Información Financiera. Navarra: Thomson-Aranzadi.

MARTíneZ, J. y G. LABATUT (2007) "La contabilización del Impuesto sobre Beneficios en el Borrador del Plan General Contable", Técnica Contable, $\mathrm{n}^{\circ} 700$, septiembre 2007, pp. 45-57.

PRICE-WATERHOUSE-COOPERS (2004) Normas Internacionales de Contabilidad 2005-2006. Desarrollo y Comentarios. Madrid: Ediciones Frances Lefebvre.

Romero FríAS, E. (Coordinador) (2008) Cambios en el PGC. Madrid: LID Editorial Empresarial S.L.

VILLARROYA LeQUERICAONANDIA, M.B. (2007) "El Impuesto sobre el Beneficio. Tratamiento en el segundo borrador del PGC", Técnica Contable, $\mathrm{n}^{\circ}$ 700, septiembre 2007, pp. 58-70. 